\title{
A Comparison Between Two Methods for Teaching Forearm Defense in Volleyball and Determining the Transfer of Learning from an Instructional Setting to a Game-Like Setting
}

\author{
Dr. Khitam Mousa Ay \\ Department of Supervision and Education, \\ Faculty of Physical Education, The University of Jordan
}

doi: 10.19044/esj.2016.v12n28p126 URL:http://dx.doi.org/10.19044/esj.2016.v12n28p126

Abstract

This study aims to make a comparison between two different methods (the Bottom-up method and the Top-down method) for teaching forearm defense in volleyball and determining the transfer of learning from an instructional setting to a game-like setting. The subjects of the study were (36) students at physical education faculty in the university of Jordan, with mean age of (20 \pm 0.5$)$ years, who were assigned in volleyball course (2) in the second semester 2015/2016. They were divided randomly into two groups: The Bottom-up method group (18) students, and the Top-down method group (18) students. Two different (4-weeks) programs were applied, three times per week for (50 minutes) by the same physical education teacher; the author of this study who is specialized in teaching volleyball, (12) lessons plans were prepared for each group in which the subjects learned the forearm defense skill and how to use this skill under game conditions. Two measurements were taken; both groups were measured at the beginning (pre-test) in order to establish that all students started at the same level of skill performance and under game-like conditions performance. After the completion of the teaching program, the final measurements were taken (post-test) to note the different impact between the two methods of teaching on the subjects performance in forearm defense skill performance and game-like conditions. Statistical analysis by using the (SPSS) program was done, included T-test for mean at pre and post test for the two groups and T-test for mean at post test between the two groups. The results showed significant differences $(p<0.05)$ in post test between the two groups in favor of the Bottom-up method group in forearm defense skill performance in volleyball. Whereas, the results showed significant differences $(p<0.05)$ in post test between the two groups in favor of the Top-down method group in transfer performance to a game-like setting. The 
study concluded that it is important to use the combination of both methods; both methods have merit according to the stage of motor learning.

Keywords: Teaching method, forearm defense, volleyball, game-like setting, skill performance

\section{Introduction}

Physical education teachers have several methods of teaching that may be employed. These methods vary in terms of who makes the decisions of what will be learned and how the learning will occur (Chatoupis \& Vagenas, 2011). Some methods are teacher-centered, whereby the teacher is the primary decision maker, and others are more student-centered allowing the students increased input on what they do (Cothran et al., 2005). It is difficult to say which style offers the maximum potential for learning because not all people will receive optimal learning by use of the same style (Thiabat, 2011). Certain material may be presented really well with one style and not as well with another. The availability of time is another concern affecting the style chosen. A teacher should always try to meet individual needs and personal differences of each student, the best methods to achieve these goals are found within the indirect, student-centered approach, the important thing is for the teacher to be able to determine what style is most appropriate in a given situation and apply it with determination and confidence. In general, learning is the gain of knowledge or skill by study, instruction, or experience (Mosston \& Ashworth 2002). Magil (1980) defined motor learning as an internal change in an individual that is inferred from the relatively permanent improvement in performance resulting from practice, Winstein and Schmidt (1990) discussed the point of view of most behavioral researchers that defines motor learning as a relatively permanent change in the underlying capability for responding, Schmidt (1991) also noted that motor learning cannot be directly measured. Learning changes are inferred from performance changes.

For motor learning to occur, learners must develop a concept of movement, they have to be able to perceive relevant environmental cues, decide what to do and when to do it, and finally produce an organized muscular movement to accomplish the desired goal (Al-Sharify \& AlZobaidy, 2014). For example, if learners wish to toss volleyball from one point to another, they must realize where the ball is coming from and where the target for the ball is, then, they must decide where to position themselves and then finally how to make contact with the ball in order to achieve the goal. Athletes' movement patterns are aimed at achieving a desired objective, if the desired objective of the movement pattern is not met, then it is altered in some manner and attempted again. 
Schmidt (1991) discussed the early history of motor learning research that led to the closed-loop theory of how movement patterns are developed, he specifically mentioned the Adams' closed-loop theory as being an important theory in its time. Adams' (1986) closed loop theory of motor learning suggested that there were two states of memory in motor learning, the memory trace and the perceptual trace. The memory trace was responsible for initiating the movement, choosing its initial direction, and determining the earliest stages of the movement, the strength of this trace is developed as a function of practice and knowledge of results. The perceptual trace is responsible for guiding the body part to the correct location, it is formed from the past experience with feedback from earlier responses and represents the sensory consequences of the body part being at the right point to perform the task. According to the Adams' closed-loop theory, when movement occurs, the learner compares the incoming feedback (from the eyes, ears, proprioceptors, etc.) against the perceptual trace to determine the correct final location of the body part. If the location is correct, the response stops; if not, a small adjustment is made and the comparison to the perceptual trace is made again until the body part is in the correct position. With increased exposure to feedback and knowledge of results, the perceptual trace is strengthened and individuals become more accurate and confident in their responding. Feedback is an important aspect in the development of motor skill according to the Adams' closed loop theory.

Scully and Newell (1985) discussed how most instructional and training programs emphasize the mastery of specific content, either knowledge or performance skills, without paying attention to the cognitive learning strategies needed to learn that particular skill. This method might help learners perform the mechanics of a specific motor skill in a controlled situation, but it does not prepare learners to use the skill in other situational contexts. Schmidt (1975) suggested there are a myriad of situations in which a specific motor skill can be used. It would be virtually impossible to teach learners how to use a motor skill such as the volleyball forearm defense in all of the possible situations it could be used. Once again; as Scully and Newell (1985) suggest, the question is how learners should be directed so that they can develop the awareness needed to perform the skill under varying conditions. Vickers (1994) outlines a dichotomy that exists in motor skill instruction; she describes two methods or models of motor skill instruction: a bottom-up method and a top-down method, she describes the bottom-up method of instruction as a model involves having learners learn a motor skill one piece at a time until the whole skill is mastered, while she describes the top-down method of instruction as a model which involves having learners learn the whole motor skill at once, without breaking it into individual 
components. Both models have different effects on motor skill acquisition, retention, and transfer.

\section{The Bottom-up method}

Bottom-up learning is the most common form of motor learning, when novice learners are taught a motor skill such as the forearm defense in volleyball in a bottom-up method, they often have difficulty transferring what they have learned when the situation becomes more dynamic. In a dynamic sport such as volleyball, there are many different cues that learners must attend to, therefore, if cognition is important to motor learning, then designing practice that encourages cognition will enhance motor learning in dynamic situations. Bottom- up theorists suggests that a motor skill is built one piece at a time until the complete skill is learned, complex skills are broken down into smaller "manageable" units, thereby creating a learning environment that allows the learner to move from one step to another in an apparently logical, sequential progression, this progression is a linear process where in parts of the skill are presented independently and sequentially, with behavioral mastery of the parts expected prior to emergence of the whole skill. Vickers (1994) discusses how the Bottom-up concept of learning has long been used as a mastery model; mastery learning is based on the premise that if learners are given appropriate instruction and enough learning time, subject mastery will occur (Bloom, 1971). The basic concept of mastery learning suggests that each separate unit or subject is mastered before moving to the next unit, mastery learning is similar to Bottom- up learning, the learning is sequential with each successive unit or subject building upon what was previously learned or mastered. Bottom-up model demands a great deal of instructor-learner contact because Bottom-up instructors manage the whole learning process, they attempt to create the environments that facilitate and support learners continuously, Bottom-up instruction in motor learning demands high emphasis on technical expertise and physical training, learners often perform the same pattern of skill over and over again until a specified degree of perfection in that skill is obtained, learners are given a tremendous amount of specific biomechanical feedback to correct errors, but they are often not given any specific time during practice to think about what they are doing. Consequently Vickers states, in the Bottom-up model of motor skill instruction, there is a low level of learner cognitive sort (Ota \& Vickers, 1999). Vickers (1994) suggests that the problem with using only the Bottom-up type of instruction is that learners can become too dependent upon the instructor for guidance. Learners using the Bottom-up method of instruction do not necessarily learn for themselves when to use their learned skills in different situations; they have learned how to use their skills only in specific controlled situations. They are dependent upon performing the skill in these situations, when the context of the situations changes, then the 
learners' performances can often deteriorate, as a result learners may acquire a degree of success in the initial stages of learning but their retention of that skill or their ability to apply the skill in a different situation is definitely weakened. Vickers (1994) suggests that learning a motor skill with the Topdown approach addresses this problem.

\section{The Top-down method}

Top-down model of instruction as described by Vickers (1994) supports a central theme of Gestalt psychology, the whole skill or concept is greater than the sum of its parts, proponents of Top-down instruction assume that learners have the ability to deal with the inherent wholeness of skills, motor skill learning with the Top-down model is the opposite of learning with the Bottom-up Model. In the Top-down model, the goal is to lead learners to perceive the whole skill or concept as quickly as possible; the learner is able to sense the big picture.Vickers (1994) describes the basic strengths of using the Top-down method of instruction for the learning of motor skills, she mentioned that in using the Top-down model of instruction individual problem solving is stressed with less instructor feedback and involvement, feedback is greatly reduced or delayed and is summative in nature, and there is often extensive use of video modeling and video feedback, the feedback is not necessarily immediate, it can be delayed, learners are encouraged to question what they are learning and are given time to detect and correct their own errors, it is more important for learners to get the "big picture" rather than to learn discrete parts of the skill, learners are asked to concentrate on the whole skill, with rhythm, timing, and visualization stressed. Top-down instructors expect learners to forge their own learning paths, the emphasis for learners is on decision making and cognitive development, the tenet of using cognitive effort to increase motor learning was supported by Shea and Morgan (1979), in their study they investigated the effect of contextual interference on the retention and transfer of a motor skill, Contextual interference in learning a skill occurs when something of a related nature interferes with what learners are trying to learn specifically. In learning a volleyball skill such as the forearm defense, contextual interference would be having to receive the ball from different positions on the court when the true skill being taught is specifically receiving the ball from one position only, Shea and Morgan (1979) discussed findings of Batting who contended that contextual interference is a major determinant of the use of multiple and variable processing strategies by individual subjects. Battig felt that contextual interference helps learners develop strategies that are appropriate for learning other material, thereby facilitating transfer. Shea and Morgan (1979) found that the results of their study suggested instructors teach a number of skills during each session for a 
number of sessions to achieve maximum retention and transfer .Shea and Morgan (1979) also mentioned that in using this type of instruction, the teacher could expect to see little progress during the early acquisition trials.

\section{The Forearm defense (Bottom-up and Top-down)}

Teaching a volleyball skill such as the forearm defense skill with the Bottom-up instructional method requires learners to move in a simple progression from a static position to a more dynamic situation (Tim \& Boris, 2006). Learners are expected to master each stage of learning before they progress to the next level, the instructor teaches arm position, leg stance, and body position, once body position, stance and arm position are learned, the teacher toss a ball gently straight to the learner, the learner is expected to forearm the pass straight back to the teacher, as the learner achieves a degree of perfection with this skill, movement is then added, which means the learner has to move forward or back right or left and receive a gently tossed ball, as the skill is mastered, more degrees of difficulty are added to it, learners have to be able to defense volleyballs coming towards them at greater speeds from further distances and a greater variety of angles, eventually, as learners achieve a specifically determined degree of success with each level of skill they put all these parts together and are then able to defense balls coming from the opposite side of the net. In learning through the Bottom-up model, learners quickly acquire a relative degree of success with the forearm defense skill; however, the following problem appears frequently when these same learners are placed in a game-like situation, although the learners have practiced the forearm defense skill repeatedly in drills, demonstrating a high degree of success in this skill, in a game-like situation their performance level on the forearm defense skill drops dramatically. Shea and Morgan (1979), Winstein and Schmidt (1990) found that learners learning motor skills by highly structured Bottom-up methods of instruction acquired a degree of success in the initial stages of learning a motor skill, these same learners, however, were not able to sustain this level of success when asked to perform the same skill under novel or unexpected conditions, the learners had to process all kinds of extrinsic information that was different from learning the specific skill of the forearm defense under controlled conditions, their skill retention of the defense decreased, the reason for this decrease may be that the skill performance was being measured under two different conditions, random versus blocked. Initially, when a motor skill is learned under Bottom-up conditions, the learning is "blocked" or structured, learners know exactly what to expect every time they are to perform the skill. In the case of the forearm defense skill, learners know the approximate speed and distance the ball is traveling towards them so that they can perform the defense appropriately, in a "random" condition 
learners are faced with a different situation while performing the forearm defense skill, learners here have to defense a smashed ball coming at them with an unknown velocity and direction, and they must compensate their movements to defense the ball appropriately. In a true form of Bottom-up learning, it would take a tremendous amount of time and repetitions for learners to learn and master all the possible combinations of defending a coming ball.

Learning a volleyball skill such as the forearm defense skill with the Top-down method is different than it is with the Bottom-up method, with the Top-down method learners might start by viewing a skilled model, receiving some simple instructions, and then taking time to practice defending the ball, they could have their practice times videotaped and then be given time to review the video and think about how to make specific corrections to their performance before trying the skill again, while feedback may be reduced or delayed, it can be variable in the Top-down method depending upon the skill level of the learner. Ota and Vickers (1999) described feedback used with the Top-down method as bandwidth feedback, in bandwidth feedback; corrections to learners are provided only when the learners' performances are outside a range of correctness, the range of correctness varies according to the skill level of the learners. The bandwidth feedback is large when learners are novices, narrowing as they progress with the skill, feedback is gradually delayed and reduced so that learners can develop confidence and learn to perform without extrinsic intervention, the bandwidth of the feedback will not be decreased until the level of performance of the learner increases to a specified level. Learning with the Top-down method of instruction gives learners a chance to develop specific meaning for the skill that they are learning. There is not a great deal of guidance or specific feedback given to the learners, Consequently, learners may take longer to achieve a degree of success in the initial stages of learning a motor skill. They will, however, develop their own specific meaning for that skill and they will not be dependent upon the instructor for guidance, when learners are not dependent upon feedback they show a greater degree of skill retention (Winstein \& Schmidt, 1990).

Vickers (1994) contrasts the differences of these two methods of instruction; she notes differences in the acquisition, retention and transfer of skill between the two methods. She discussed the results shown in the instruction, practice and feedback literature in cognition and motor learning that show an opposite learning effect between these two methods. Vickers (1994) refers to this learning effect as the reversal effect, she calls it the reversal effect because of the way in which subject performance changes from acquisition to retention in the learning of a skill. Subjects trained under highly structured and fully assisted (Bottom-up) method achieve greater 
success during acquisition but falter later during retention and transfer trials. Conversely subjects trained under less structured, less assisted (Top-down) method show the opposite profile, They achieve lower levels of performance during acquisition but higher levels during later transfer and retention, the subjects trained under the Top-down method therefore exhibit more longterm learning (Ota \&Vickers, 1999). If the ultimate goal of learning is to retain knowledge and skill, then the Top-down model may be the most appropriate method of motor skill instruction. Şirinkan and GŸndoğdu (2011) noted that the research supporting this effect is controversial, but if there is validity for the Top-down method of instruction, then it would have an interesting impact on the pedagogy of motor learning.

This study aims to examine and compare the Top-down method versus the Bottom-up method of instruction in teaching the volleyball forearm defense skill in novice learners to determine which method of motor skill instruction the Bottom-up or the Top-down method would provide the greatest skill acquisition and raise the level of transfer of learning of the forearm defense skill in volleyball in novice learners from an instructional setting to a game-like setting.

\section{Method}

This study compared the effects of two different teaching methods, the Bottom-up method and the Top-down method, upon the learning of the forearm defense skill in volleyball by novice learners to determine which method of motor skill instruction would provide the greatest skill acquisition for the novice learners and raise the level of transfer of learning of the forearm defense skill in volleyball from an instructional setting to a gamelike setting. The subjects were (36) students at physical education faculty in the university of Jordan, with mean age of $(20 \pm 0.5)$ years, who were assigned in volleyball course (2) in the second semester 2015/2016. They were divided randomly into two groups: The Bottom-up method group (18) students, and the Top-down method group (18) students. Two different (4weeks) programs were applied, (3)times per week for (50 minutes) by the same physical education teacher; the author of this study who is specialized in teaching volleyball, (12) lessons plans were prepared for each group in which the subjects learned the forearm defense skill and how to use this skill under game conditions. Both quantitative and qualitative results were generated through pre and post tests. In the pre test, both groups went through physical subjective performance evaluations which measure the level of their forearm defense skill performance and under game like conditions to check that there were no differences among all subjects. 


\section{Procedure and Participants}

Permission to implement the program for this study was secured from the head dean of physical education faculty at the University of Jordan, as well as from the students as subjects of the study. After completing the pretests for both groups, two different methods programs was given by the same physical education teacher; the author of this study who is specialized in teaching volleyball,(12 lessons) plans were prepared and applied for each one of the teaching methods, The teaching program took (4-weeks), (3) times per week, for (50 minutes) per session. All subjects of both groups were taught the forearm defense skill in volleyball, the first group subjects were instructed using a Bottom-up method, and the second group subjects were instructed using a Top-down method. The instruction sessions and all testing sessions were held in the volleyball indoor court in the faculty of physical education at the University of Jordan. The teaching sessions for the two different methods of instructions were held on the same days (Sundays, Tuesdays, Thursdays) on different times, the Top-down instructions at (9$9.50 \mathrm{am})$ and the Bottom-up instructions at (11-11.50 am). The sessions were developed with the tenets of each method of instruction in mind, the Bottomup sessions were progressive in nature, going from the basics to a controlled game situation, while the Top-down sessions started with the whole skill being used in simple game-like drills progressing to more dynamic game-like drills, the instructor used as much feedback as he wanted when dealing with the Bottom-up group subjects, while she made her feedback general with the Top-down group subjects, and gave them time to actually think about how they were performing the skill., after completing all the teaching sessions for both methods of instructions the post-test was done.

\section{Bottom- up group}

In the Bottom-up method of instruction, the forearm defense skill was broken down into its individual components, the components were then taught to the subjects in a progression that allowed them to build on each component until the whole skill was mastered, the sessions progressed from the basics of the forearm defense skill to the point where subjects started to use the skill in a controlled game. Each session is scheduled to last approximately 50 minutes, with the Bottom-up method constant correction and feedback is an integral part of the instruction process, Instructor uses lots of feedback, correction and encouragement.

\section{The Basic technique}

-The platform angle: standing position (upper body and lower body), hands position, arms position, arm distance from chest. 
-Contact point: Keeping eyes on the ball, moving to save the ball, lateral movement right to save the ball(review lead foot), lateral movement left to save the ball (review lead foot), move forward and to right to save the ball, move forward and to (left) to save the ball,

-Body ball relationship (acceleration): starting with saving gentle tossed balls ending with saving hard spiked balls (straight, to right, to left, start behind baseline and run towards net - stop to save dropped balls, start at net and move backwards - stop to save spiked hard balls

-Resulting trajectory and always call mine: To prevent collisions with players and waste the ball.

\section{Top-down group}

In the Top-down method of instruction, the subjects were taught the whole skill of the forearm defense, they were given time to think about how best to perform the skill to achieve the desired result, the sessions were scheduled to last approximately 50 minute, the learners were strongly encouraged to find their own way of learning the skill, feedback was occasional and very general in nature, and it was a wide bandwidth-very general with time given for the learners to think about what was said so that they may develop a meaning for how to accomplish the forearm defense skill.

\section{The Basic technique}

- The platform angle.

- Contact point.

- Body-ball relationship (acceleration).

- Resulting trajectory and always call mine.

-Drills in this method while not necessarily static will start simple and work to more complex as the skill level of the learners is determined.

-The instructor makes a model; she had the assistant toss a ball, so that the subjects can observe, the instructor performed the forearm defense correctly.

-Every learner made the forearm defense and count good ones, try and figure out why some were better than others.

-The instructor asked the learners what you would do to make the forearm defense better.

-The instructor made a simple reminder telling the learners: "make contact on this part" of the forearm defense.

- Start to introduce "game" (decision making) concept, learners are required to learn their skill in the environment in which it is supposed to be used.

- Learners work in groups of four, playing a volleyball game with regular scoring and rally points, they should be ready to move, find a way to save the ball and keep it off the floor (game to 10 switch foursome). 
-The instructor gave Cues: Where is it easiest to defend the ball from? (ball in front left or right?).

-The instructor reminds the learners "What did you do that allowed you to defend the ball and get it to where you wanted it to go."

-More game like drills (as much of a game as their skill level allows), keep scoring for good forearm defense skill, instructor gives the learners a chance to find what works, feedback is minimal and general, instructor keeps encouraging the learners to find out what works best for them to save the ball and get it where it is supposed to go.

\section{Measurements}

The quantitative results were obtained by having the subjects' skill in the forearm defense rated by two professional volleyball judges observing a videotape of the subjects' performance in the pre-test and the post-test and game like conditions, the two judges worked together to devise a rating system that would determine the score on the different skill components of the forearm defense skill, the video tape performance was viewed separately by both judges. Judges were blind to whether subjects had participated in the Top-down or Bottom-up instruction; the ratings of the judges were then averaged to determine the rating in the particular skill component of the forearm defense skill for each subject. Individual subject performance ratings were scored for the four skill points of the forearm defense, each individual subject score was then converted to a percent for comparison among subjects, test conditions and teaching methods, and the differences in the acquisition, and transfer of skill from an instructional setting to a gamelike setting between the two methods . An overall combined score mean was then determined for each condition and each teaching method. Both groups had their forearm defense skill levels assessed before beginning Instruction and four weeks after completing instructions, by having their performances videotaped, each subject of both groups saved ten balls from a controlled source, the controlled source was the instructor standing on the opposite court six meters away from each subject, and passing the ball over the net to each subject, each subject was graded on his performance on four skill points of the forearm defense skill: platform, contact point, body-ball alignment and acceleration, resulting trajectory, these skill points were selected by the researcher because they are considered critical for the proper execution of the forearm defense skill in volleyball. Each subject performed ten trials for the forearm defense skill and all trails were videotaped for further analysis by two observers who were trained and were experts in volleyball, they watched the videotapes which were collected for this study and grades were recorded in the recording sheet. 
Post-instruction for forearm defense skill performance was obtained differently, but all subjects of both groups were measured the same way, the difference in measuring skill performance between the pre-test and the post-test was done to determine if there was any transfer of learning from an instructional setting to a game-like setting, It was thought that novice players having little experience in the sport of volleyball would have difficulty performing the forearm defense skill in a dynamic game-like situation. After completing the instructions, they would have enough experience to perform the forearm defense skill under more dynamic conditions , based on this assumption, all subjects in the pre-test condition were tested in a static, controlled situation, in order to measure the transfer of skill to a more game-like situations, the subjects were tested in a dynamic, game-like setting, in the post-test where decision making was important. In the post-test, the subjects had their performance videotaped again, while performing in a controlled game of 4 versus 4 , each forearm defense subjects performed in the game was evaluated, the scoring system for the evaluation was identical to the system used in the pre-test condition and the four individuals on the court rotated their positions during their time on the court, and the actual groups played several games during the testing session. This rotation was done to maximize the number of forearm defense skill performed by each subject, scoring of skill level of the forearm defense skill was done by two volleyball performance experts who were trained in the method of scoring the skill points of the forearm defense skill. The scorers were blinded, in that they did not know from which instruction group students came. The individual scores for each student in the condition under which they were measured were averaged from the two scorers to obtain a total score in the forearm defense skill. This created a set of scores for pre-instruction and post-instruction.

\section{Results and discussion}

This study compared the effects of two different teaching methods, the Bottom-up and the Top-down method, upon the learning of the forearm defense skill in volleyball by novice learners to determine which method of motor skill instruction would provide the greatest skill acquisition for the novice learners and raise the level of transfer of learning of the forearm defense skill in volleyball from an instructional setting to a game-like setting, both quantitative and qualitative results were generated. 
Table 1. Means, SD and T-test for checking the equivalence between the two groups for forearm defense skill performance in pre-test.

\begin{tabular}{|c|c|c|c|}
\hline $\begin{array}{l}\text { Teaching group } \\
\text { skill pre-test }\end{array}$ & M & SD & t-test \\
\hline $\begin{array}{c}\text { Bottom - up } \\
\mathrm{N}=18\end{array}$ & 15.56 & 5.55 & \multirow[t]{2}{*}{0.45} \\
\hline $\begin{array}{c}\text { Top - down } \\
N=18\end{array}$ & 14.67 & 6.24 & \\
\hline
\end{tabular}

Table (1) indicates that the calculated t values were not significant between the two groups in forearm defense skill performance. The probability values supports that there were no significant differences between the two groups at 0.05 level concluding that the two groups are equivalent.

Table 2. Means, SD and T-test for checking the equivalence between the two groups in

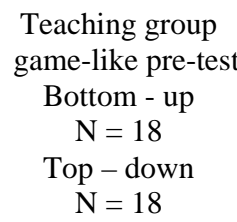
game-like in pre-test.

Table (2) indicates that the calculated t values were not significant between the two groups in game-like. The probability values supports that there were no significant differences between the two groups at 0.05 level concluding that the two groups are equivalent.

Table 3. Means, SD and T-test for forearm defense skill performance between the two

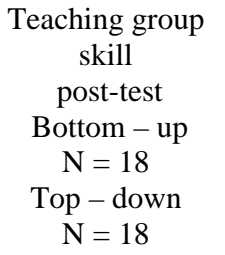
groups in post-test.

Table (3) shows the values of means, standard deviations and calculated t between the two groups for forearm defense skill performance in the post tests. The Bottom-up method post-test mean was 29.78 with standard deviation 6.17. While the Top-down method post test mean was 23.06 with standard deviation of 7.89 and the calculated t value 2.84 .

Table 4. Means, SD and T-test for game-like between the two groups in post-test.

$\begin{array}{ccccc}\begin{array}{c}\text { Teaching group } \\ \text { game-like post-test }\end{array} & \mathrm{M} & \mathrm{SD} & \text { t-test } & \text { Sig } \\ \begin{array}{c}\text { Bottom }- \text { up } \\ \mathrm{N}=18\end{array} & 25.17 & 5.62 & & \\ \begin{array}{c}\text { Top }- \text { down } \\ \mathrm{N}=18\end{array} & 30.56 & 6.63 & 2.65 & * 0.013 \\ & & & & \end{array}$


Table (4) shows the values of means, standard deviations and calculated $t$ between the two groups for game-like in post test. The Bottomup method post-test mean was 25.17 with standard deviation5.62. While the Top-down method post test mean was 30.56 with standard deviation of 6.63 and the calculated t value 2.65.

Table 5. Means, SD and T-test for forearm defense skill performance between the two groups in post test.

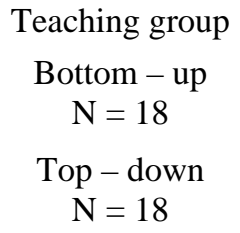

\begin{tabular}{ccc} 
Skill test & M & SD \\
\hline Pre & 15.56 & 5.55 \\
\hline Post & 29.78 & 6.17 \\
\hline Pre & 14.67 & 6.24 \\
\hline Post & 23.06 & 7.89
\end{tabular}

t-test Sig $6.74 * 0.000$ $* 0.002$

Table (5) shows the values of means, standard deviations and calculated $t$ between the pre and post tests in each group for forearm defense skill performance. The Bottom-up method pre test mean was 15.56 with standard deviation 5.55, while the post test mean was29.78 with standard deviation of 6.17; the calculated t value was 6.74 indicating that means differences were significant in favor of the post test. While the Top-down method pre test mean was 14.67 with standard deviation 6.24 , while the post test mean was 23.06 with standard deviation of 7.89; the calculated t value 3.70 indicating that means differences were significant in favor of the post test. Significant differences were found between the pre- and post-test scores in the (forearm defense skill performance) for both groups.

The post-test scores provided evidence that both groups showed better performance in the forearm defense skill than the pre-test. The posttest scores also provided evidence that the Bottom-up method group had better performance than the Top-down group in the forearm defense skill; this means that learning the skill through the Bottom-up method is more effective than learning the same skill through the Top-down method. The author attributes this result to the high emphasis on technical expertise and physical training to every small unit of the skill while using the Bottom-up method in teaching the forearm defense skill. This is consistent with Tim and Boris (2006) who noted that In Bottom-up method subjects performed the same pattern of the skill over and over again until a specified degree of perfection in the skill is obtained. The researcher also returns this result to the great deal of instructor-learner contact and the continuous feedback which was used in Bottom-up method, this is consistent with Al-Rabiey and Ameen 2011) who mentioned that in Bottom-up motor learning method learners are given a tremendous amount of specific biomechanical feedback to correct performance errors, but they were not given any specific time during practice to think about what they are doing. While in using the Top- 
down method individual problem solving is stressed with less instructor feedback and involvement, feedback is greatly reduced or delayed and learners are encouraged to question what they are learning and are given time to detect and correct their own errors, the author of this study thinks that subjects who learned through the Top-down method with less feedback and who corrected their own errors, took more time to master the skill, this is the reason why subjects in the Bottom-up method had better performance. AlKhalaf (2007) noted that breaking down the skill into smaller changeable units creates a learning environment that allows learners to move from one step to another in an apparently logical, sequential progression, this progression is a linear process wherein parts of the skill are presented independently and sequentially, with behavioral mastery of the parts expected, prior to emergence of the whole skill. The Bottom-up learning method is similar to mastery learning, both are based on the premise that if learners are given appropriate instruction and enough learning time, the motor skill mastery will occur, the basic concept of the Bottom-up learning method and the mastery learning suggests that each separate unit of the motor skill is mastered before moving to the next unit and the learning is sequential, with each successive unit building upon what was previously learned or mastered.(Bloom,1971).

Table 6. Means, SD and T-test differences for game-like between the two groups in post

Teaching group
$\begin{gathered}\text { Bottom }- \text { up } \\ \text { N = } 18 \\ \text { Top - down } \\ N=18\end{gathered}$
test.

Table (6) shows the values of means, standard deviations and calculated t between the pre and post tests in each group for game-like condition. The Bottom-up method pre test mean was 15.83 with standard deviation 6.35. While the post test mean was 25.17 with standard deviation of 5.62; the calculated t value was 4.42 indicating that means differences were significant in favor of the post test. While the Top-down method pre test mean was 16.67 with standard deviation 6.22 , while the post test mean was 30.56 with standard deviation of 6.63 ; the calculated t value 5.88 indicating that means differences were significant in favor of the post test. Significant differences were found between the pre- and post-test scores in the (game-like) for both groups. Both groups showed improvement.

The post-test scores provided evidence that both groups showed better performance in game-like condition than the pre-test. The post-test scores also provided evidence that the Top-down method group had better 
performance than the Bottom-up method in game-like conditions. The author thinks that although the Bottom-up method subjects had better scores in the skill performance than the Top-down method subjects because they performed the same pattern of the forearm defense skill over and over again until a specified degree of perfection in the skill is obtained, they were given a tremendous amount of specific biomechanical feedback to correct performance errors, but they were not given any specific time during practice to think about what they are doing. Consequently Ota and Vickers (1999) stated, in the Bottom-up method of motor skill instruction, there is a low level of learner cognitive sort, When novice learners are taught a motor skill such as the forearm defense in a Bottom-up method, they often have difficulty transferring what they have learned when the situation becomes more dynamic. While Al-Sharify and Al-Zobaidy (2014) noted that in a dynamic sport such as volleyball, there are many different cues that learners must attend to, therefore, if cognition is important to motor learning, then designing practice that encourages cognition will enhance motor learning in dynamic situations. Vickers(1994) mentioned that the problem when using only the Bottom-up method of instruction is that learners become too dependent upon the instructor for guidance and feedback, they don't necessarily learn for themselves when to use their learned skills in different situations,

The author of this study noticed that subjects who learned through the Bottom-up method, have learned how to use their skills only in specific situations, they were dependent upon performing in these situations, when the situations changed their performance become weak this is consistent with what Al-Khalaf (2006) noted, that when learning through the Bottom-up method, learners work under controlled situations, when the context of the situations changes, then the learners performances deteriorate, they may acquire a degree of success in the initial stages of learning, but their ability to apply the skill in different situation is weakened. Gallahue and Cleland (2003) agree with Vickers (1994) who suggests that learning a motor skill with the Top-down method addresses this problem, because learners in the Top-down method are asked to concentrate on the whole skill, with rhythm, timing, and visualization stressed, they are expected to forge their learning paths, and the emphasis for learners is on decision making and cognitive development. The tents of using cognitive effort to increase motor learning was supported by Shea and Morgan (1979) study which investigated the effect of contextual interference on the retention and transfer of a motor skill, contextual interference in learning a skill occurs when something of a related nature interferes with what learners are trying to learn specifically. In learning a volleyball skill such as the forearm defense, contextual interference would be having to defense the ball from different positions on 
the court when the true skill being taught is specifically defending the ball from one position only, contextual interference helps learners develop strategies that are appropriate for learning other material, thereby facilitating transfer. Evidence suggests (Thiabat, 2011; Mosston \& Ashworth, 2002 ; Winstein \& Schmidt, 1990;Shea \& Morgan, 1979) that the Top-down method is the best model for the teaching of motor learning, It allows for better cognitive development and therefore better skill retention and transfer, the problem is that skill acquisition suffers when only the Top-down method of instruction is used.

In conclusion, in learning through the Bottom-up method of instruction, subjects of this study quickly acquired a relative degree of success with the forearm defense performance in volleyball. However, the following problem appears frequently when these same subjects are placed in a game-like situation, although they have practiced the forearm defense repeatedly in drills, demonstrating a high degree of success in this skill, but in a game-like situation their performance level on the forearm defense drops dramatically. On the other hand, learning with the Top-down method of instruction gave the subjects a chance to develop specific meaning for the skill they were learning, there was not a great deal of guidance or specific feedback given to the subjects consequently, they took longer time to achieve a degree of success in the initial stages of learning the forearm defense, they developed their own specific meaning for the skill, they were not dependent upon the instructor for guidance, they were not dependent upon feedback, they showed greater degree of skill in game-like situation .

This study suggests that strong guidance is needed for beginners to learn how to properly perform a complex skill performance such as the forearm defense skill in volleyball, specifically playing in a game-like situation does not direct learners towards proper skill performance (Nesser \& Demchak, 2007). Ota and Vickers (1999), who support the Top-down method, suggested the importance of the combination of both methods, both methods have merit, however, the Bottom-up method is important, when dealing with novice learners, special populations, or individual learners where repetition of skills or physical training is needed, while the Top-down method preserves much of what is valuable in the Bottom-up method, the Top-down method takes learners forward so that there is more balance between the technical, mental, physiological and social foundations of performance. Each level of learner brings a different focus for the instructor. As novice learners, they require a great deal of guidance and feedback to ensure they can attend to the proper information and acquire the proper body movements to perform the skill. Buck and Bryce (1990) study suggested the need for structure when teaching novice learners, their study, looked only at achievement and did not determine whether or not a specific method of 
instruction, such as the Top-down method, would increase achievement. More advanced learners or experts, who have already acquired the ability to perform the skill, would need to be able to adapt the motor skill to varying conditions, advanced learners, however, are not the same as novice learners (Harrison \& Blakemorce, 1989).

\section{Conclusion}

The question for this study was which method of motor skill instruction, the Bottom-up method or the Top-down method, is best for novice learners to acquire the forearm defense skill performance in volleyball for later retention and transfer to game-like conditions. The study concluded that it is important to use the combination of both methods; both methods have merit according to the stage of motor learning.

\section{References:}

1. Adams, J. (1986). Use of the model's knowledge of results to increase the observer's performance, Journal of Human Movement Studies, 12, 89-98.

2. Al-Khalaf. M. (2007). The impact of using the partial and integrated in teaching table tennis basic skills, published research, the second international scientific conference (Scientific development in sports and physical education, The Yarmouk University, Irbid, Jordan.

3. Al-Khalaf. M. (2006). The effect of using random and sequential training in learning some of closed and opened skills in squash, published research, The fifth international scientific conference, Vol 3, Faculty of physical education, The University of Jordan, Amman, Jordan.

4. Al-Rabiey,M \& Ameen, S.(2011). Methods of teaching physical education, publisher of scientific books, Lebanon, Bayrot.

5. Al-Sharify, W \& Al-Zobaidy, Q. (2014). The effect of using individual and cooperative and command teaching methods in teaching basic skills in football, published research, Dirasat Journal, Vol 33, No2, The university of Jordan.

6. Bloom, B. (1971). Mastery learning theory and practice, New York: Holt, Rinehart \& Winston.

7. Buck, M \& Bryce, G. (1990). An analysis of learning trails and their relationship to achievement in volleyball, Journal of teaching in physical education, 10,134-152.

8. Chatoupis, C \& Vagenas, G. ( 2011) An analysis of published process-product research on physical education teaching method, International Journal of Applied Sports Sciences, 23 (1) , pp. 271289. 
9. Cothran et al., (2005) A cross-cultural investigation of the use of teaching styles Research Quarterly for Exercise and Sport, 76 (2005), pp. 193-201.

10. Gallahue, D \& Cleland, F. (2003). Developmental physical education for all children. USA: Human Kinetics.

11. Harrison, M \& Blakemorce, L. (1989). Instructional strategies for secondary school physical education, Dubuque, IA: Brown.

12. Magil, R. (1980). Motor learning concepts and applications, Dubuque, IA: Brown.

13. Mosston, M \& Ashworth, S. (2002). Teaching physical education. (5th ed). NY: Benjamin Cummings.

14. Nesser, T \& Demchak, T. (2007). Variations of preseason conditioning on volleyball Performance, Journal of Exercise Physiology Online, 10(5), 35-42.

15. Ota, D \& Vickers, J. (1999). The effects of variable practice on the retention and transfer of two volleyball skills in male club level athletes, International journal of volleyball research, (1), 18-24.

16. Schmidt, R. (1991). Motor learning and performance: From principles to practice, Champaign, IL: Human kinetics.

17. Scully, D \& Newell, K. (1985). Observational learning and the acquisition of motor skills: toward a visual perception perspective. Journal of Human Movement Studies, 11, 169-186.

18. Shea, J \& Morgan, R. (1979). Contextual interference effects on the acquisition retention, and transfer of a motor skill, Journal of experimental psychological: Human learning and Memory, 5(2), 179187.

19. Şirinkan, A \& G Ÿndoğdu, K. (2011). The perceptions of teachers in relation to physical education curriculum and instructional plans. Elementary Education Online, 10 (1), 144-159 .

20. Thiabat, M. (2011). The effect of problem solving method on improving some skills of table tennis among physical education student in Yarmouk University, Journal of united Arab Universities.

21. Tim, J \& Boris, G . (2006) the development of a standardized skill assessment for junior volleyball players, International Journal of Sports Physiology and Performance, 1:95-107 .Human Kinetics, Inc, pp 99.

22. Vickers, J. (1999). Instructional Design for Teaching Physical Activities. Champaign, IL: Human Kinetics Books.

23. Vickers, J. (1994). Psychological research in sport pedagogy: exploring the reversal effect, sport science review, 1(1), 28-40. 
24. Winstein, C \& Schmidt, R. (1990). Reduced knowledge of results enhances motor skill learning, Journal of experimental psychology: Learning, memory and cognition, 16(4), 677-691. 